\title{
SOLAR OBLATENESS AND DIFFERENTIALLY \\ ROTATING POLYTROPES
}

\author{
G. G. FAHLMAN, M. D. T. NAYLOR and S. P. S. ANAND \\ David Dunlap Observatory, University of Toronto, Canada
}

Dicke and Goldenberg (1967a) measured the solar oblateness to be $\sigma=(5 \pm 0.7) \times 10^{-5}$ and subsequently interpreted this measurement as evidence that the solar interior rotates with a period of 1.8 . With this interpretation, they then showed that the observed oblateness causes an $8 \%$ discrepancy in the Einstein prediction of the perihelion advance of Mercury. The stability analysis of Goldreich and Schubert (1967) seems to preclude such a fast rotation of the solar interior although magnetic field effects could alter their conclusions (Dicke, 1967). More recently Goldreich and Schubert (1968) and Fricke (1969) have calculated upper bounds to the solar oblateness essentially by finding the steepest distribution of angular velocity that is consistent with secular stability at each point in the equatorial plane of the sun. Fricke's result of $\sigma_{\max }=1.4 \times 10^{-5}$ is based on a stronger stability criterion than that of Goldreich and Schubert who found $\sigma_{\max }=1.4 \times 10^{-4}$; Fricke, however, suggests that this may be in error and should actually be $\sigma_{\max }=3.4 \times 10^{-5}$. In their calculations of $\sigma_{\max }$ the above authors assumed that the outer convective layers of the sun are rotating uniformly and that the angular velocity in the interior is a function of the radial distance from the center of the sun only. We note that while these assumptions are reasonable, neither of them is supported by the observed solar rotation.

Detailed calculations based on uniformly rotating polytropic models led Anand and Fahlman (1968) to suggest that a modest differential rotation extending throughout the entire sun may explain the observed oblateness without implying a significant change in the calculated perihelion advance of Mercury. We report here the results of some calculations based on applying the differential rotation law of Stoeckly (1965) to solar polytropes.

The Stoeckly differential rotation formula is

$$
\omega=\omega_{0} \exp \left(-c s^{2} / R^{2}\right),
$$

where $\omega_{0}$ is the central angular velocity, $s$ is the cylindrical radial coordinate measured from the axis of rotation, $R$ is the solar equatorial radius and the constant parameter $c$ determines the amount of differential rotation. If the Rayleigh stability criterion, $\partial / \partial s\left(\omega s^{2}\right) \geqslant 0$, is to be everywhere satisfied then we must have $c \leqslant 1$. Rayleigh's criterion can be stabilized by a suitable gradient of mean molecular weight (Goldreich and Schubert, 1967) so that we may expect $c>1$ in the evolved solar interior.

In our calculations we have assumed that the Stoeckly law can be applied throughout the entire sun, including the convection zone. This assumption will limit the usefulness of our results because the Stoeckly law does not give the observed latitude variation in angular velocity on the solar surface. The equator of the sun is observed to be 
rotating faster than the layers at higher latitudes whereas the Stoeckly law implies that the equator is rotating slower than the pole by a factor of $e^{-c}$. We may however assume differential rotation throughout the convection zone since it appears that a modest differential rotation can be supported by an anisotropic convective viscosity (Cocke, 1967). If this theory is applicable we would generally require that the anisotropy be a function of both radius and latitude in order to give the angular velocity both a radial latitude dependence. In view of the large differences in mixing length and convective viscosity the convection zone such a possibility can not be excluded.

The perihelion advance of Mercury, $\Delta \bar{\omega}$ radians per orbital period, caused by the mass quadrupole moment of the rotationally distorted sun can be written (Anand and Fahlman, 1968)

$$
\Delta \bar{\omega}=\frac{3(C-A)}{M} D .
$$

Here $C$ and $A$ are the principal moments of inertia of the sun in the equatorial plane and along the rotation axes, $M$ is the solar mass and $D$ is a quantity which depends only on the elements of Mercury's orbit. If the outer layers of the sun are uniformly rotating then it can be shown that $\sigma$ is analytically related to $(C-A)$ and hence to the perihelion advance. For non-uniform rotation, this is not the case and we must calculate $(C-A)$ independently of $\sigma$ to obtain $\Delta \bar{\omega}$.

The models have been obtained by employing the technique devised by Stoeckly (1965) which gives accurate results for both fast uniform rotation and the restricted type of non-uniform rotation given by Equation (1). The Stoeckly scheme replaces the differential equations by difference equations which are then linearized and the solution is obtained by iteration. The actual dependent variable is the difference between two successive approximations to the physical variable. Critical uniformly rotating models were obtained (Naylor, 1968) for the polytropic indices 1.5, 2.0 and 3.0. The results agree with those of James (1964) to better than $1 \%$.

In the present work we have required that the dimensionless equatorial angular velocity of the models be equivalent to the solar value (Allen, 1963). The first model in each series was static and provided a reference for the differentially rotating models which were obtained by increasing the parameter $c$ from $c=0$ in steps of 0.05 . The double integration that is necessary to obtain the value of $C-A$ was accomplished by using an eleven point Gauss-Legendre quadrature for the angular dimension and a repeated application (at 40-50 points) of Simpson's rule for the radial dimension. The final results should be accurate to a few percent or better.

In Table I we give values of the oblateness, $\sigma$ and the perihelion advance, $\Delta \bar{\omega}$, now measured in sec of arc per century, for various values of $c$ and for three different polytropicindices, $n$, which should bracket the solar structure. In general for the same value of $c$, polytropes with less mass concentration are more oblate and have higher quadrupole moments than those which are more centrally condensed. For the case $n=3$, the maximum oblateness consistent with the Rayleigh stability criterion is $3.54 \times 10^{-5}$. Further calculations for this case show that we require $c=1.25$ to obtain 
TABLE I

Oblateness and perihelion advance

\begin{tabular}{lllllll} 
& $n=2.5$ & & \multicolumn{2}{c}{$n=3.0$} & & \multicolumn{2}{c}{$n=3.25$} & \\
\cline { 4 - 7 } & $\sigma \times 10^{-5}$ & $\Delta \bar{\omega}$ & $\sigma \times 10^{-5}$ & $\Delta \bar{\omega}$ & $\sigma \times 10^{-5}$ & $\Delta \bar{\omega}$ \\
\hline 0.85 & 3.11 & 0.23 & 2.92 & 0.10 & 2.84 & 0.06 \\
0.90 & 3.31 & 0.24 & 3.15 & 0.11 & 3.02 & 0.06 \\
0.95 & 3.50 & 0.26 & 3.31 & 0.11 & 3.33 & 0.07 \\
1.00 & 3.81 & 0.28 & 3.54 & 0.12 & 3.46 & 0.08 \\
\hline
\end{tabular}

$\sigma=5 \times 10^{-5}$. This value of $c$ corresponds to the centre rotating 3.5 times faster than the equator. In all cases the perihelion advance of Mercury due to the rotational distortion is entirely negligible.

Thus we have at least shown that it is possible for a stellar surface to be rotating slowly at the equator and still have a fairly large oblateness but a small mass quadrupole moment. These results are not directly applicable to the sun because of our use of the Stoeckly law as discussed earlier. Nevertheless, they do not contradict the results of Dicke and Goldenberg (1967b) who used a simple perturbation technique to show that the effect of differential rotation on the solar surface is unimportant. In their perturbation analysis it is assumed that the angular velocity function has approximate spherical symmetry in the outer layers and can be represented as a two-term Legendre expansion. If the angular velocity law deviates from spherical symmetry so that the higher order terms in the Legendre expansion are not negligible, as is the case for the Stoeckly law, their analysis is no longer applicable. However, in view of the observed solar rotation law, it is difficult to see how the case discussed here can be relevant to the sun. We can only conclude that if the solar oblateness is to be explained by differential rotation then we must have a non-spherical rotation law but to find such a law that is also compatible with the observed solar rotation appears to be very difficult.

\section{Acknowledgements}

This research was supported in part by the National Research Council of Canada and the Department of University Affairs of the Province of Ontario.

\section{References}

Allen, C. W.: 1963, Astrophysical Quantities, Athlone Press, London.

Anand, S. P. S.: 1968, Astrophys. J. 153, 135.

Anand, S. P. S. and Fahlman, G. G.: 1968, Icarus 8, 492.

Chandrasekhar, S.: 1933, Monthly Notices Roy. Astron. Soc. 93, 390.

Cocke, W. J.: 1967, Astrophys. J. 150, 1041.

Dicke, R. H.: 1967, Science 157, 960.

Dicke, R. H. and Goldenberg, H. Mark: 1967a, Phys. Rev. Letters 18, 313.

Dicke, R. H. and Goldenberg, H. Mark: 1967b, Nature 214, 1294.

Fricke, K.: 1969, Astrophys. Letters 3, 219. 
Goldreich, P. and Schubert, G.: 1967, Astrophys. J. 150, 571.

Goldreich, P. and Schubert, G.: 1968, Astrophys. J. 154, 1005.

James, R. A.: 1964, Astrophys. J. 140, 552.

Naylor, M. D. T.: 1968, unpublished.

Stoeckly, R.: 1965, Astrophys. J. 142, 208.

\section{Discussion}

Dicke: The rotating model considered by Mr. Fahlman has a negligibly small gravitational quadrupole moment, and the oblateness is determined wholly by rotation at the surface. Two different models with the same surface rotation but different internal rotations will have the same oblateness if their quadrupole moments are negligibly small. Mr. Fahlman's models give more rapid rotation at the pole and are not applicable to the sun. For the observed rotation the corresponding oblateness is $0.81 \times 10^{-5}$. 